\title{
Large Titanosaur from Indo-Pakistan Peninsula
}

\author{
Muhammad Sadiq Malkani \\ Geological Survey of Pakistan, Muzaffarabad, Pakistan \\ Email:malkanims@yahoo.com
}

How to cite this paper: Malkani, M.S. (2019) Large Titanosaur from Indo-Pakistan Peninsula. Open Journal of Geology, 9, 635-638.

https://doi.org/10.4236/ojg.2019.910061

Received: August 16, 2019

Accepted: September 21, 2019

Published: September 24, 2019

Copyright (c) 2019 by author(s) and Scientific Research Publishing Inc. This work is licensed under the Creative Commons Attribution International License (CC BY 4.0).

http://creativecommons.org/licenses/by/4.0/

\begin{abstract}
Indo-Pakistan subcontinent is lucky to host the smallest, medium and large sized titanosaurian sauropod dinosaurs. Saraikimasoom is the smallest sized, and Gspsaurus is the medium sized transversely stocky, Pakisaurus and Isisaurus are the large sized titanosaurs of South Asia. Pakisaurus balochistani is a slender type of pakisaurid titanosaurian based on associated vertebral and appendicular skeletons. Pakisaurus balochistani includes key elements like transversely thin or narrow tibia and ventrally not reduced tall caudal vertebrae, along with other elements which are significant for cladistic analysis.
\end{abstract}

\section{Keywords}

Large Titanosaur, Latest Maastrichtian, Vitakri Lameta Formation, Indo-Pakistan

\section{Introduction}

Pakisaurus is included among four recently recognised titanosaurian sauropods from South Asia. Pakisaurus balochistani is described here and significant for comparisons, Gondwanan paleobiogeography and evolutionary studies.

\section{Pakisaurus balochistani Large Titanosaur from Indo-Pakistan}

Systematic paleontology of Pakisaurus balochistani is as follows. Dinosauria, Saurischia, Sauropoda, Titanosauria, Pakisauridae [1], Pakisaurus [1], and Pakisaurus balochistani [1] (Figure 1). Pakisaurus balochistani as a new genus and new species was formally published by Malkani [1]. Pakisaurus balochistani holotypic caudal vertebrae [1] and lectotypic vertebral and appendicular elements from South Kinwa 4 holotypic and lectotypic locality (Figure 1), and referred fossils from West Bor 2, northern Top Kinwa 16, North Alam 19n, Shalghara 3, Mari Bohri 15, Darwaza 8 and Grut 9 localities are found in the latest Maastrichtian 


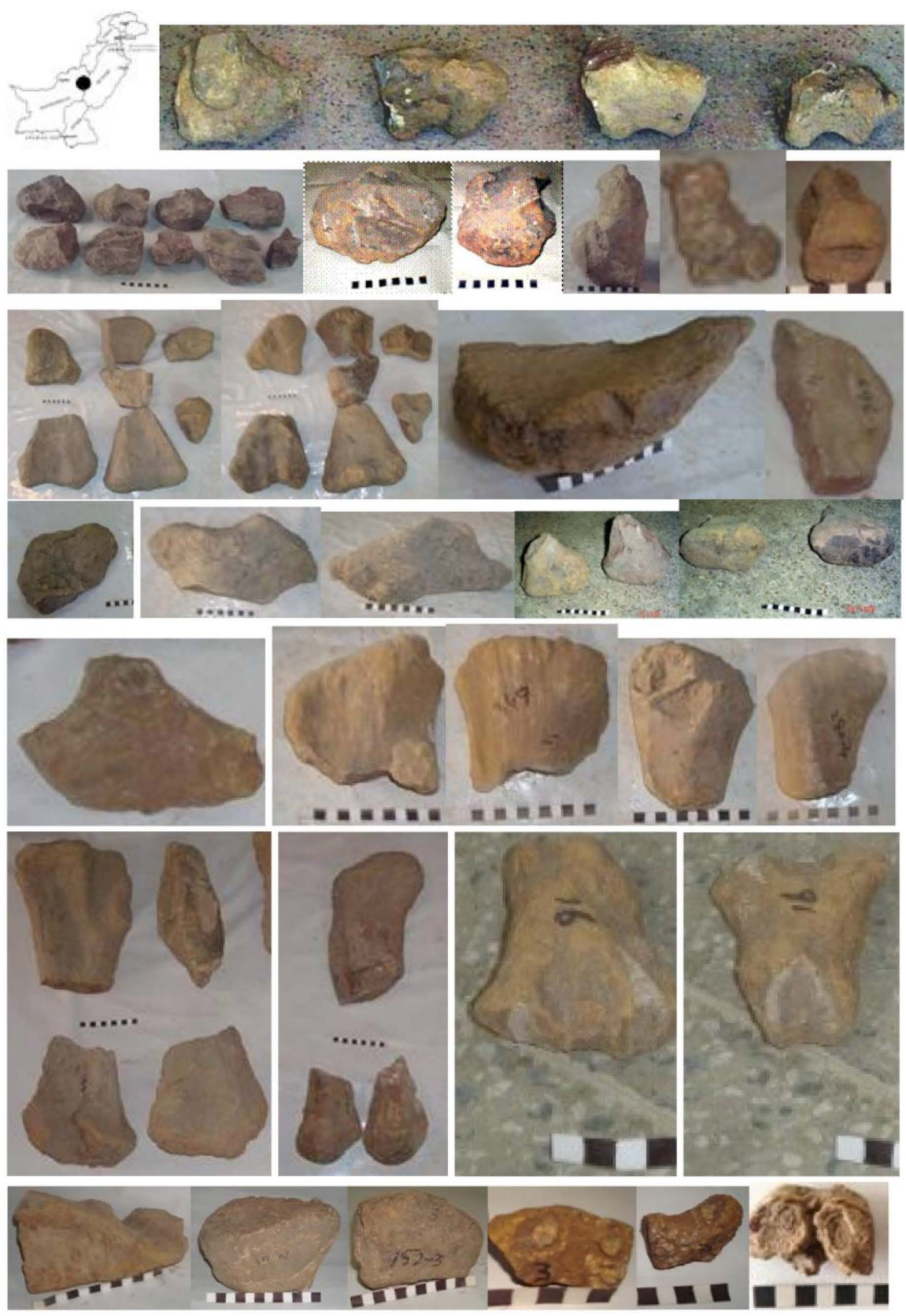

Figure 1. Pakisaurus balochistani fossils. Row 1, Map of Pakistan (black circle) show Kinwa type locality, holotypic caudal vertebrae from Kinwa. Row 2, 3, 4, 5, lectotypic vertebral and appendicular elements from Kinwa. Row 6, referred associated tibia and humerus (photo 1/p 1) and femur (p 2) from west Bor or lower Bor; chevron 2 views ( 3 - p 4). Row 7, referred coracoid (p 1), proximal radius (p 2), ungual (p 3 ) and osteoderms jaw ramus with diastema type embedded spikes (p 4 - p 5) and dentary symphysis type with teeth like spikes ( $\mathrm{p} \mathrm{6)}$. Scale, each black or white digit is $1 \mathrm{~cm}$.

(67 - 66 Ma ago) Vitakri Lameta Formation of Fort Munro Group, Barkhan District, Balochistan, central Pakistan. These fossils are housed in the museum of Geological Survey of Pakistan, Quetta. Some fossils from Vitakri Lameta Formation of India are also referred. Genus Pakisaurus honour the host country Pakis- 
tan, saurus mean lizard. Species named $P$. balochistani honour the host province Balochistan.

\subsection{Diagnosis of Pakisaurus balochistani Large Titanosaur}

Pakisaurus balochistani sharing with Titanosauria as procoelous caudals; forward insertion of neural arches on caudals; prominent olecranon process on ulna and vertebrae lacking hyposphene-hypantrum articulations Pakisauridae characters are same as genus and species. Pakisaurus balochistani is recognised as more derived lithostrotian titanosaur based on procoelous anterior and middle caudal vertebrae. Pakisaurus balochistani is recognised as most derived Poripuch lithostrotian titanosaur based on procoelous anterior, middle and posterior caudal vertebrae. Pakisaurus balochistani is characterised by long slender legs and tall tail; narrow, long and recurved teeth with constant thickness from base to tip (except tip); large sized braincase with D-shaped occipital condyle; basal tubera breadth narrower than occipital condyle; caudals are tall quadrangular shape except a few anteriormost caudals which are broad; distal most caudal centrum anterior articular face shape procoelous while posterior face ball is biconvex cone with a horizontal transverse groove in the middle; distal scapula relatively less expanded than Gspsaurus, distal scapula with relatively anteroposteriorly long glenoid; distal scapular articular length for coracoid is relatively small (unlike Isisaurus); acromian process is narrow; expanded radial condyle exposed on the anterior aspect of distal humerus (unlike Isisaurus); femoral shaft, lateral margin shape, proximal one-third deflected medially with wavy style (and not straight as in Isisaurus); transversely narrow proximal Tibia with arc shaped lateral fibular condylar ridge ended just below cnemial crest; and osteoderms spikes on ramus.

\subsection{Description of Pakisaurus balochistani Large Titanosaur}

Teeth are narrow, long and recurved with constant thickness from base to tip (except tip). Braincase is large sized. Occipital condyle is large and broad and greater part of its convexity faces downward. Thick occipital condyle has broad flat upper surface. Basal tubera breadth is narrower than occipital condyle. Occipital region of skull is flat and distally recurved. Paroccipital processes oriented transversely. Basal tubera are long process, diverging laterally with relatively lower angle than Gspsaurus who have high angle basal tubera. Basal tubera directed slightly backwards. Basipterygoid processes diverge somewhat anteriorly and much longer than the basal tubera. Caudals are tall quadrangular shape except a few anteriormost caudals which are broad. Anteriormost caudals neural spine is vertical. Distalmost caudal centrum anterior articular face shape procoelous while posterior ball face is biconvex cone with a horizontal transverse groove in mid. Distal scapula relatively less expanded than Gspsaurus. Distal scapula has relatively anteroposterior long glenoid. Articular length for coracoid is relatively small (unlike Isisaurus). Acromian process of scapula is narrow. 
Proximal humerus has prominent lateral process than medial process. Expanded radial condyle exposed on the anterior aspect of distal humerus (unlike Isisaurus). Proximal ulna with tri-limbs has prominent olecranon process. Femoral shaft, proximal one-third deflected medially with wavy style (and not straight as in Isisaurus). Proximal Tibia is transversely thin bone or narrow (unlike thick lense shape of Gspsaurus and thick subcircular shape of Saraikimasoom) with arc shaped fibular condylar ridge ending just below the cnemial crest. Posterior process of proximal tibia is about half of total anteroposterior width in Pakisaurus, while posterior process is about three quarter 3/4 of the total anteroposterior proximal width of Isisaurus tibia. Ratio of anteroposterior width of distal end to anteroposterior width of proximal end is 0.80 in Pakisaurus while the ratio of anteroposterior width of distal end to anteroposterior width of proximal end is as low as 0.64 in Isisaurus. Osteoderms present, arced ramus with diastema having random false teeth like spikes on ramus and also on base skin which forms diastema on arced ramus and false dentary type symphysis with spikes/spines; and Pedal ungual sickle-shaped, much deeper dorsoventrally than broad transversely.

\section{Acknowledgements}

This work is a contribution to UNESCO/IUGS/IGCP 679 project.

\section{Conflicts of Interest}

The author declares no conflicts of interest regarding the publication of this paper.

\section{References}

[1] Malkani, M.S. (2006) Biodiversity of Saurischian Dinosaurs from the Latest Cretaceous Park of Pakistan. Journal of Applied and Emerging Sciences, 1, 8-140. 